\title{
Hazardous Gas Detection and Alerting Using Sensors
}

\author{
Akshaya Priya s \\ UG Student,Department of CSE \\ S.A Engineering College \\ Chennai-600116 \\ Keerthana M \\ UG Student,Department of CSE \\ S.A Engineering College \\ Chennai-600116
}

\author{
Jenifer M \\ UG Student,Department of CSE \\ S.A Engineering College \\ Chennai-600116
}

\author{
Prasanna Kumar R \\ Associate Professor,Department of CSE \\ S.A Engineering College \\ Chennai-600116
}

\begin{abstract}
Home safety has been a major issue in our day-to-day life and it is everyone's duty to provide good safety system at home. The main objective is to eradicate hazardous gas from home using IoT. The Internet of Things(IoT) is a system of interrelated computing devices which has the feature of providing unique features without human-to-human or human-to-computer interaction. This project is aimed at developing the home safety against the leakage of LPG gas. In case of any leakage it provides an SMS alert to the user using the GSM and switch off the gas knob or valve. In addition it also turnoff the power supply and inverter automatically in order provide a complete home safety.
\end{abstract}

\section{Keywords}

Alerting, Gas detection, Gas sensor, GSM module, Safety, Security.

\section{INTRODUCTION}

In the earlier days people made use of the natural stove using wood and fire which in order created a lot of smoke resulted in air pollution. In order to reduce the air pollution people replaced the natural stove which is a traditional method into a LPG gas stove which again hike the level of the air pollution. This is because LPG gas results in gas leakage and due to this many fire accidents in the world and this results in many death of the people. One of the most remarkable fire accidents due to gas leakage at bhopal. LPG is the Liquified Petroleum Gas which is a easily flammable gas as it contains propane $\left(\mathrm{C}_{3} \mathrm{H}_{8}\right)$ or butane $\left(\mathrm{C}_{4} \mathrm{H}_{10}\right)$.

The sensors are used to monitor the leakage of the gas as sensor plays as important role in our day-to-day life. Sensors are of different kinds and are present in the different fields such as monitoring the health of old patients, sensing the environment, leakage of gas etc. Today every individual is living in a smart world and our entire house is based on automation system in order to prevent accidents and safeguard ourself.

The leakage of the gas is monitored periodically in order to avoid accidents because about people aged below 60 of the people can smell the leakage but the people who is aged equal and above 60 are having the smell disorder as the gas is odourless, tasteless and invisible. This is a real time project which is implemented in order to save the valuable lives of many people.

This system always monitors the gas cylinder and if there is a leakage of gas then it can perform the necessary safety measures accordingly within a second that is within a twinkle of eye. It can also perform its task even when an individual is not present at home or in an vacation.

As the technology has been increasing day by day the sensors is very sensible even when it encounters a very small leakage of gas as the prevention is always better than cure. The system used is very advanced as there would not be any wrong indication and even if there is any power cutoff situation this system can also work in battery and take the necessary actions accordingly.

In the earlier times of 1980 and 1990, the gas leakage detection system was done by introducing a chemically infused paper in gas and if the paper changes its color then there is a gas leakage. If the leakage of gas is not monitored then it leads to suffocation and later consequences is explosion.

The leakage of gas must be controlled as it is commonly used in home for cooking. More number of research papers are introduced but still it produces a wrong indication and some projects are really expensive but this method is very simple and available at a very cheaper rate so that it can be introduced in every individual house as lives are more important than anything.

\section{SURVEY ON HAZARDOUS GAS DETECTION AND ALERTING SYSTEM}

PoojaBhamare, ShivanjaliDalvi, ManasiBhamare, DipaliDube, MansiBhonsale[1], the paper focuses on the leakage of gas at car, home etc. If there is any leakage of gas then the exhaust fan will automatically turned on, an alarm will be produced and a gas leakage message will be sent to the customer.

This project can be used in every places and if the system sense any leakage of gas then it results in providing an visual indication on the concentration of gas and it also contains an alarming unit which provides alarm during the leakage of gas. It provide a quick response time.

Falohun A.S, Oke A.O, Abolaji B.M and Oladejo O.E[2], the paper focuses on, detecting the leakage of the gas and in case of any leakage of gas it provides an alarm to the consumers and in addition it also switch off all the electrical appliances at home. This system uses MQ-9 sensor to detect the leakage of gas. 
This system is designed in such a way that it is very flexible and can be implemented at cheaper rates. This project was designed in such a way that it eliminates the common error and the maintenance process is also very simple.

Prof. K.R.Katole, VrushaliBagade, BhagyashreeBagade, AnkitaSoni and HiranmayeeKamde[3], the paper focuses on, the toxic gases which was sensed using arduino sensor and in case of any leakage of gas it will provide an concentration of gas leakage in the LCD display. The concentration will be displayed in percentage.

This project deals with a microcontroller which receives the signals from the sensor and then it sends the corresponding signal to the devices which is connected externally. In addition to it this system is a less complex and can be done very quickly.

Prof. M.Amsaveni, A.Anurupa, R.S.AnuPreetha, C.Malarvizhi and M.Gunasekaran,[5], the paper focuses on, detecting the LPG gas leakage automatically and if there is any leakage of gas then the gas valve is closed automatically and power supply is also cut off. In addition it also send an SMS alert to the user.

This project deals with detecting the gas leakage and automatically controlling the concentration of the gas leakage by turning off the power supply and closing the valve.

HrushnkeshKeluskar, PoojaChavan, Sonalikudale, G.D.Salunke, SatishPawar[6], the paper focuses on gas leakage and fire accidents at home. This project provides an SMS alert to the consumer if it detects an leakage of the LPG gas.

This paper provides cheaper as well as accurate solution to prevent and detect gas leakage and fire accidents by sending a message to consumer, activating buzzer and also by opening automatic window. In order to provide complete safety at home MQ-7 sensor is used. This project is a simple system which decreases the fire accidents, gas leakage and also used to prevent the fault which occurs at home.

T.Soundarya, J.V.Anchitaalagammai, G.DeepaPriya and S.S.KarthickKumar[8], the paper focuses on detection, monitoring and control system of LPG leakage. The stove knob is automatically controlled by using relay DC motar and also rebooking of the cylinder when the level of gas goes below the normal weight of cylinder.

The system provides control action by closing the regulator knob, after that the system sends an alert message to the users and the fire station within short time of the leakage.

K.PadmaPriya, M.Surekha, R.Preethi, T.Devika and N.Dhivya[9], the paper focuses on, detecting the gas leakage and sends an SMS alert to the consumer and also the power supply will be turned off automatically. In addition to that the level of the gas in the cylinder will be monitored and if the level of the gas of thecylinder is low then the user is intimated about the issue and the cylinder is booked automatically which in order saves time.

This project deals with the automatic booking of the cylinder when the concentration level of gas in cylinder is low. This system provides a simple technique and it is also available at a low cost.

HitendraRawat, AshishKushwah, KhyatiAsthana, AkankshaShivhare[10], this paper focuses on developing the home security against intruders, gas leakage and fire. In case of any above incidents happened this system sends an alert message to the consumer. This system consists of a background into the area of 8051 microcontroller and communication through mobile which are interfaced with each other and AT commands are used for the communication.

This project is a simple system which can be applied as a safety in home, car from gas leakage. This project is very simple and also can be applied to detect the alcohol level in individual. It provides the perfect result and it is a error free method.

S.Rajitha and T.Swapna[12], the paper focuses on the detection of the LPG gas leakage and sends an SMS message to the user. This system also monitors the concentration of the leak and also displays the concentration in the LCD display. If the level of the concentration is more than the normal level then an alarm is generated at home and exhaust fan is turned on in order to reduce the concentration of the leakage of gas.

This paper concludes that it can be used in many areas. This system easily detects the leakage of the gas and provide the necessary details to the consumer via the SMS if the concentration is more than the normal level.

V.Ramya and B.Palaniappan[13], the paper focuses on, detecting the leakage of the LPG gas and the concentration of the LPG gas is displayed in the LCD display and if the concentration is more than the normal level then an alert message is sent to the consumer immediately.

This project deals with the detection of various types of the gases and this system is very sensitive and provide a fast response time. The LCD display shows the concentration of the leakage of the gas and the system monitors the concentration accordingly.

M.B.Frish, R.T. Wainner, B.D.Green, M.C.Laderer and M.G.Allen[15], the paper focuses on the system that uses trace sensing technology and also detects the leakage and in addition this project can also be used to sense the environment. This method made use of a new leakage detection tool which is an alternative to the traditional leakage detection method.

This project concludes that it uses a modern tool as it is very simple and less complex. In addition it also provide the project at a very low cost.

Young Jin Jung, Yang Koo Lee, Dong Gyu Lee, Yongmi Lee, Silvia Nittel, Kate Beard, Kwang Woo Nam, Keun Ho Ryu[14], the paper focuses on the system that monitors the environment regarding the air pollution and if there is any change in the environment that whether the concentration of the air pollution is more than the normal level then it immediately provides an alarm as an alert

This project concludes that it monitors the area where the human intervention is very poor and it provides the necessary safety measures for making the environment free from the pollution. This system is very simple and available at a very low cost. The sensor are used in a more efficient way in order to reduce the charging of battery.

M.S.Kasar1, RupaliDhaygude, SnehalGodse, SnehaGurgule[4], the paper focuses on monitoring the gas cylinder whether there is a leakage of gas and if it encounters any leakage of gas then it provide the necessarysafety measures to solve it. In addition it also helps in booking the gas when it encounters that the concentration is below the normal level.

This project concludes that it helps the individual to perform their jobs without giving concentration to small things as the system itself helps to automatically refill the gas cylinder and provide a complete safety to the people at home.

S Shyamaladevi, V G Rajaramya, P Rajasekar, P Sebastin Ashok[11], the paper focuses on monitoring the gas cylinder whether the concentration is below the normal level and it make uses of ARM-7 to automatically refill the gas cylinder. In addition it also provides complete safety by detecting the leakage of the gas and provides an SMS alert to the user.

This project deals with the detection of the gas and if it encounters any gas leakage then it sends SMS alert to the consumer immediately. In order it also book the gas cylinder automatically 
when it becomes empty and it do all the process without the human interaction.

Arun Raj, AthiraViswanathan, Athul T.S[7], the paper focuses on supervising the gas cylinder whether there is a emission of gas and if so there is any exhalation of gas then it sends an immediate alert to the user using GSM module. In addition it also supervise the concentration of gas level and if it is below the usual level then immediately sends an alert to the user.

This project concludes that it is a very straightforward method and available at a very low cost. It look for the leakage of gas and takes the necessary measures to eradicate it. In addition it also show the level of concentration and sends an SMS alert to the user about the booking.

\section{PROPOSED SYSTEM}

The proposed system illustrates that when the sensor encounters the LPG gas leakage then the sensor which is fixed at the cylinder knob will turn off the knob automatically and the alert message is sent to the consumer and finally the power is turned off automatically. In case if the power has cut down already due to some reasons then the gas sensor can work with the help of the battery. In some cases where the system supervises the leakage of the gas and if there is an leakage then it cut off the power supply but still the generator operates and the power has turned on then this system automatically shut down the inverter power supply.

\subsection{SYSTEM OPERATION}

In this project MQ-6 gas sensor plays an major role. The MQ-6 sensor is used for detecting the leakage of the LPG gas. It can easily measure the concentration of the gas leakage from $200 \mathrm{ppm}$ to $1000 \mathrm{ppm}$. In that an heater is fixed which makes the sensitive components in the circuit to work.

The MQ-6 gas sensor usually contains 6 pins in it. In that 6 pins, the 4 pins are used to receive the signals and the remaining pin is made used by the heater.

When there is a leakage of gas then the voltage at the output pin of the MQ-6 is increased and it monitors the concentration of the LPG gas. If the concentration of the leakage of the gas is more than the normal level then an SMS alert message is sent to the consumer immediately using the GSM module. The Atmega89 is used to provide the necessary details to the stepper motor to rotate completely $90^{\circ}$ in order to turnoff the gas knob and immediately cutoff the power supply even if the individual is not present at the home.

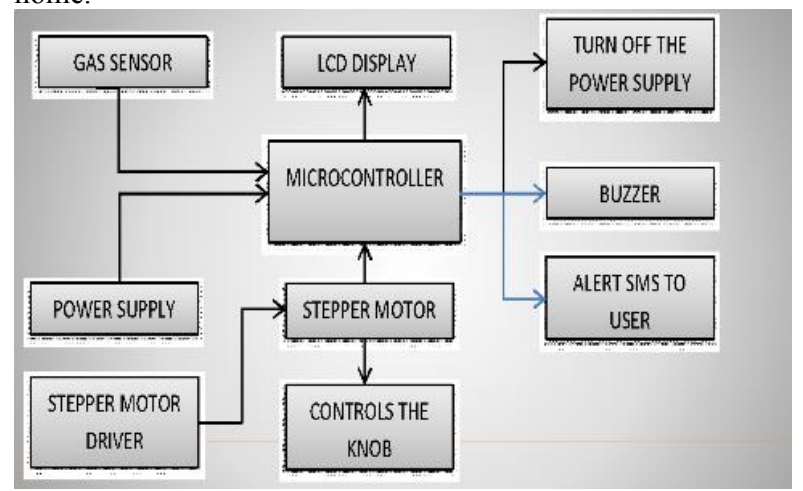

Figure 1: Block diagram for user-interactive gas detection and prevention system.

\subsection{FLOW CONTROL}

The gas sensor sense whether there is a leakage of gas and if there is a gas leakage and if the concentration is more than the normal level then relay is activated and then the power is turned off. In addition it also rorate the stepper motor to 90 degree in order to turn off the gas knob and also checks whether the user is within the range of transmission if so the SMS alert is sent to the consumer if not the SMS alert is sent to the user family member. If the power cut is done already due to some reasons then the system can work in battery and it immediately turn off the inverter as soon as the main power supply is cut down.

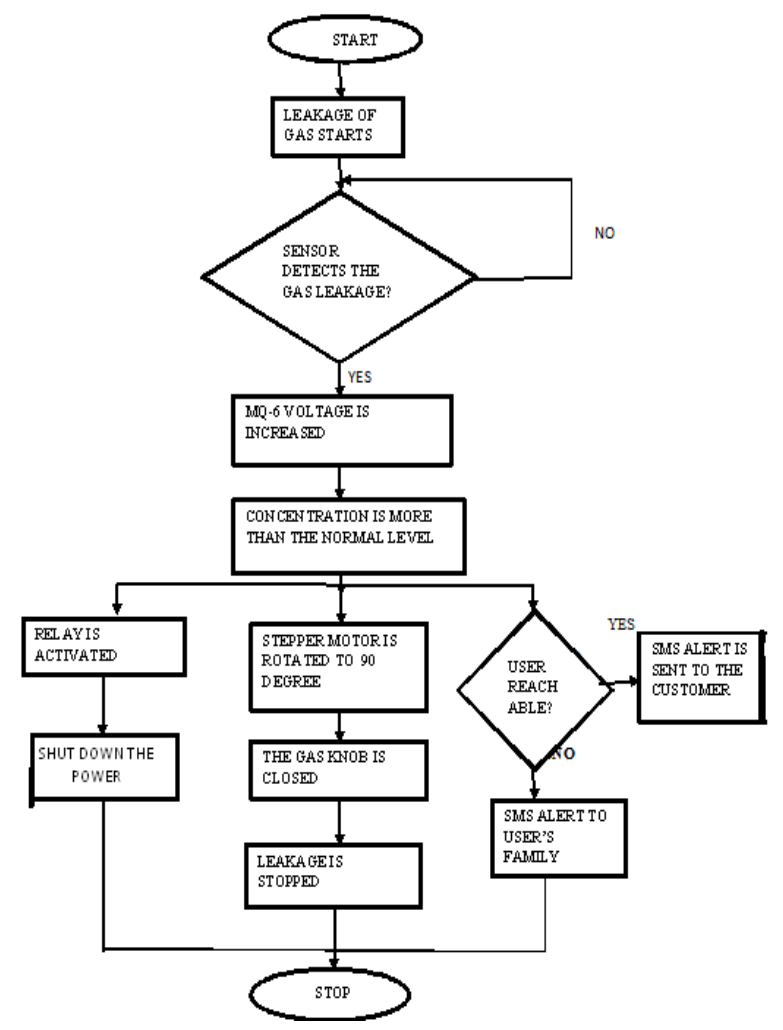

Figure1.1 Flow of gas detection and alerting system

\subsection{HARDWARE}

\subsubsection{ATMEGA}

ATMEGA is a AVR architecture which provides numerous instructions that can be executed in a clock signal identical.

It consists of features like high performance and low power design etc.

\subsubsection{BUZZER}

Buzzer is also known as Beeper. It is a signal which can be available in an audio format. In addition to it generates signal in an acoustic format. The uses of buzzer are alarm devices, timers. The types of Buzzers are electrochemical, piezoelectric, mechanical. The buzzer is used to indicate a light when a particular key is pressed and intimate the user using alarm. 


\subsubsection{STEPPERMOTOR DRIVER}

Stepper motor drive is otherwise known as Stepper motor driver. It is actually a circuit which operates a stepper motor. It acts as an interface to the motor. The components are controller, Driver IC, power supply .

\subsubsection{LPG GAS SENSOR}

LPG/CNG gas sensor displays the level of LPG in a visual format. It can sense the toxic gases easily. The gas sensor has its own conductivity and its conductivity increases when there is an increasing concentration of the leakage of the gas. It is compatible for the gases like propane or butane.

\subsubsection{RESISTORS}

Resistor accelerates the electric power in a circuit. It provides voltage for the electric device.

\subsubsection{CAPACITORS}

Capacitor stores energy in the electrical device in a dual terminal. It is based on physical structure of conductors and dielectric material.

\subsubsection{DIODE}

Diode is a single directional current flow with respect to voltage. In an electrical device it operates like an valve

\subsubsection{STEPPER MOTOR}

Stepper motor is also known as stepping motor or step motor. It bisects the entire rotation into equal steps. It consists of steps without any feedback sensor. It is based on speed and torque. The stepper motor can be commonly denoted as a step by step rotation. When the sensor detects the leakage of the gas it makes the stepper motor to rotate upto 90 degree in order to close the cylinder knob.

\subsubsection{GSM MODULE}

Global System for Mobile is the expansion of the GSM. The main objective of the GSM is communication. When the sensor senses the LPG gas leakage the GSM immediately sends an alert message to the consumer if the user is in the reachable area.

\subsection{SOFTWARE}

The software specification which is used in this project arearduino compiler and Mc programming language that is embedded C.

\subsubsection{Arduino compiler}

The arduino compiler generally means the workspace where an individual can write their own code. Some of the common commands used are verify,new,open etc.

\subsubsection{Embedded C}

Embedded $\mathrm{c}$ is a programming language which has been evolved from a $\mathrm{C}$ language. The syntax of Embedded $\mathrm{C}$ is very similar to $\mathrm{C}$ programming language. The embedded $\mathrm{C}$ contains all the positive features of $\mathrm{C}$ and in addition it contains some additional special features which includes some special header files. The flow of the program is similar for both Embedded $\mathrm{C}$ and $\mathrm{C}$ programming language. Embedded $\mathrm{C}$ is compatible for both 8 bit processor and 64 bit processor.

\section{REFERENCES}

[1]. PoojaBhamare, ShivanjaliDalvi, ManasiBhamare, DipaliDube, MansiBhonsale, "A Survey on GasBo for LPG Gas Detection using Mobile", published in 2016.

[2]. Falohun A.S, Oke A.O, Abolaji B.M and Oladejo O.E, "Dangerous Gas Detection using an Integrated Circuit and MQ9", published in 2016.

[3]. Prof. K.R.Katole, VrushaliBagade, BhagyashreeBagade, AnkitaSoni and HiranmayeeKamde, "Hazardous Gas Detection using ARDUINO", published in 2016.

[4]. M.S.Kasar1, RupaliDhaygude, SnehalGodse, SnehaGurgule,"Automatic LPG Gas Booking and Detection System", published in 2016.

[5].Prof.M.Amsaveni,A.Anurupa,R.S.AnuPreetha， C.Malarvizhi and M.Gunasekaran, " Gsm based LPG leakage detection and controlling system", published in 2015.

[6]. HrushnkeshKeluskar, PoojaChavan, Sonalikudale, G.D.Salunke, SatishPawar, "GSM Based Home Safety", published in 2015.

[7]. Arun Raj, AthiraViswanathan, Athul T.S, "LPG Gas Monitoring System", published in 2015.

[8].T.Soundarya, J.V.Anchitaalagammai, G.DeepaPriya and S.S.Karthick Kumar, "C-Leakage: Cylinder LPG Gas Leakage Detection for Home Safety”, published in 2014.

[9]. K.PadmaPriya, M.Surekha, R.Preethi, T.Devika and N.Dhivya, "Smart gas cylinder using Embedded system", published in 2014.

[10]. HitendraRawat, AshishKushwah, KhyatiAsthana, AkankshaShivhare, "LPG Gas Leakage Detection and Control System", published in 2014.

[11]. S Shyamaladevi, V G Rajaramya, P Rajasekar, P Sebastin Ashok, "ARM7 BASED AUTOMATED HIGH PERFORMANCE SYSTEM FOR LPG REFILL BOOKING \& LEAKAGE DETECTION" published in 2014.

[12]. S.Rajitha and T.Swapna, "A Security alert system using GSM for gas leakage", published in 2012.

[13]. V.Ramya and B.Palaniappan, "Embedded system for Hazardous Gas detection and Alerting”, published in 2012.

[14]. Yongmi Lee, Young Jin Jung, Kwang Woo Nam, Silvia Nittel, Kate beard, Keun Ho Ryu, "Geosensor Data Representation Using Layered Slope Grids", published in 2012.

[15]. M.B.Frish, R.T. Wainner, B.D.Green, M.C.Laderer and M.G.Allen, "Standoff gas leak detectors based on tunable diode laser absorption spectroscopy", published in 2005. 